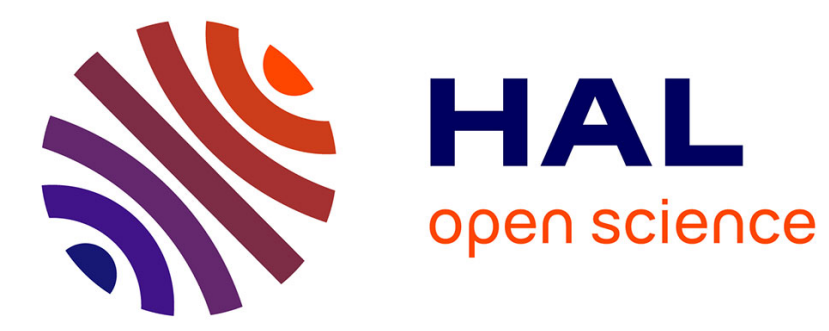

\title{
Interplay between JSRV, an oncogenic retrovirus and the pulmonary epithelium
}

Fabienne Archer, Jean-François Mornex, Caroline Leroux

\section{To cite this version:}

Fabienne Archer, Jean-François Mornex, Caroline Leroux. Interplay between JSRV, an oncogenic retrovirus and the pulmonary epithelium. Current Immunology Reviews, 2012, 8. hal-02642718

\section{HAL Id: hal-02642718 \\ https://hal.inrae.fr/hal-02642718}

Submitted on 28 May 2020

HAL is a multi-disciplinary open access archive for the deposit and dissemination of scientific research documents, whether they are published or not. The documents may come from teaching and research institutions in France or abroad, or from public or private research centers.
L'archive ouverte pluridisciplinaire HAL, est destinée au dépôt et à la diffusion de documents scientifiques de niveau recherche, publiés ou non, émanant des établissements d'enseignement et de recherche français ou étrangers, des laboratoires publics ou privés. 


\title{
Interplay Between JSRV, an Oncogenic Retrovirus, and the Pulmonary Epithelium
}

\author{
Fabienne Archer*, Jean-François Mornex and Caroline Leroux
}

\author{
UMR754 Rétrovirus et Pathologie Comparée, Université de Lyon, Université Lyon 1, INRA, VetAgroSup, Ecole \\ Pratique des Hautes Etudes, IFR128 BioSciences Gerland Sud, Hospices Civils de Lyon, Lyon, France
}

\begin{abstract}
Jaagsiekte sheep retrovirus (JSRV) is a retrovirus which infects small ruminants and is responsible for a natural lung cancer. This virus bears an oncogenic envelope inducing epithelial cell transformation from the deep lung. The cells, main target of the infection and constitutive of the tumors, are alveolar type II cells in the alveoli and Clara cells in the bronchioli. The immune response to JSRV infection is poorly understood. The specific humoral response is limited and the cell-mediated response is marked by CD4+ lymphocytopenia, neutrophilia, and macrophage invasion of the tumor. The mechanisms of viral immune evasion could be explained by the direct and indirect immunosuppressive effects of the virus, and by the immune tolerance of the infected hosts due to the presence in the sheep genome of endogenous viral forms (enJSRV).
\end{abstract}

Keywords: Lung, JSRV, immune response, adenocarcinoma, alveolar type II cells.

The airway epithelium being strategically positioned at the interface with the environment, it plays a key role in the host defence system. A large volume of air is inhaled every day and the extensive surface area of the lung makes the respiratory system especially vulnerable to airborneinfectious agents such as influenza viruses, respiratory syncitial virus, rhinovirus, coronavirus and retroviruses. We will focus here on JSRV, a retrovirus infecting the lung parenchyma and inducing an adenocarcinoma in sheep.

\section{VIRAL IMMUNE RESPONSE IN THE LUNG}

The innate immune response is the first line of defense against viral pathogens. It requires their early detection through pathogen-specific recognition receptors and recruitment of efficient antiviral effectors (extensively reviewed in [1-4]). Upon pathogen infection, the lung epithelium responds by releasing antimicrobial peptides into the airway lumen, chemokines and cytokines into the submucosa that initiate an inflammatory response and induce the recruitment of phagocytes, dendritic cells and lymphocytes. Cells of the innate immune system express PRRs (Pattern Recognition Receptors) able to regulate the synthesis of antiviral type I and III interferons (IFNs) and pro-inflammatory cytokines $[5,6]$. PRRs that participate in viral sensing include toll-like receptors (TLR), RNA helicases and cytosolic DNA sensors [4, 7]. Toll like receptors located on cell surfaces and/or in cytoplasm act as initiators of the innate immune response by providing the ability for the host to recognize PAMPS (PathogenAssociated Molecular Patterns). Intracellular TLRs and other PRRs such as RNA helicase molecules (i.e. retinoic-acid inducible protein 1 [RIG-1] and melanoma differentiation-

*Address correspondence to this author at the UMR754 Rétrovirus et Pathologie Comparée, Université de Lyon, Université Lyon 1, INRA, VetAgroSup, Ecole Pratique des Hautes Etudes, IFR128 BioSciences Gerland Sud, Hospices Civils de Lyon, Lyon, France;

Tel: ?????????????????; Fax: ???????????????????;

E-mail: fabienne.archer@univ-lyon1.fr sentinel and epithelial cells, are able to detect intracellular dsRNA (double strand RNA) and ssRNA (single strand RNA) to initiate type I IFN and pro-inflammatory cytokine production via activation of specific-transcription factors including IRFs (Interferon Regulatory Factors ) and NF- $\mathrm{kB}$ (Nuclear Factor-kappaB).

In recent years, it has become clear that airway epithelial cells not only constitute a mechanical barrier, but also actively contribute to the innate immune system [7]. Although there have been numerous studies on viral infection of the conducting airways (reviewed in [8]) relatively few have focused on the distal gas-exchange unit of the deep lung, site of severe clinical infection such as SARS-CoV (severe acute respiratory syndrome-coronavirus) or avian influenza virus. The epithelium where gas exchanges occur is constituted of two main cell types. Alveolar type I cells are flat terminally-differentiated cells that cover about $95 \%$ of the alveolar surface area and are mainly involved in gas exchange. Cuboidal alveolar type II cells are metabolically active, produce pulmonary surfactant and transport fluid and sodium to the basolateral surface to keep the alveolar surface suitable for gas exchange [9]. Alveolar type II cells are self-renewing and are able to transdifferentiate into alveolar type I cells when they need to be replaced. Both alveolar type I and type II cells participate in the innate immune response by producing interferons and cytokines in response to virus infection $[4,10,11]$. They also produce antimicrobial substances such as surfactant protein A (SP-A), surfactant protein-D (SP-D), beta-defensins or lysozyme in response to microbial infection $[7,12,13]$. SP$\mathrm{A}$ and SP-D interact with the glycoconjugates and lipids on the virus surface inducing aggregation of complexes, enhancement of phagocytosis and release of cytokines and chemokines at the site of infection [14]. Beta-defensins have a dual role in antiviral activity : they may block viral infection by directly acting on virions or they may affect the target cell and thereby indirectly interfere with viral infection [15]. Taken together, all these mechanisms take place in order to eliminate viruses from the lung and maintain the 
integrity of the alveolar function. Some viruses have developed strategies to bypass or delude this defence in order to initiate cell deregulation or even tumours.

\section{OVINE PULMONARY ADENOCARCINOMA: A RETROVIRUS-INDUCED LUNG CANCER}

JSRV (Jaagsiekte Sheep RetroVirus) is a $\beta$-retrovirus, infecting small ruminants. This virus is responsible for a pulmonary adenocarcinoma associated with the transformation of epithelial cells from the lung parenchyma, i.e. alveolar type II cells in the alveoli and Clara cells in the bronchioli [16] (Fig. 1). The disease is usually sporadic within flocks but can be epizootic in some specific conditions of farming, leading to the death of up to $5 \%$ of the animals [16]. This animal tumour is clinically, radiologically and histologically related to the pneumonic form of human bronchioloalveolar cancer (pBAC), a rare form of lung tumour in humans which represent less than $4 \%$ of all adenocarcinomas [16, 17]. These similarities have always intrigued pathologists and chest physicians and have been stressed as early as $1939[18,19]$. Both the pneumonic form of human lung adenocarcinoma and the ovine pulmonary adenocarcinoma are described as mixed-type adenocarcinoma, associating a predominance of bronchioloalveolar lesions and papillary and/or acinar lesions. Ovine pulmonary adenocarcinoma has been considered as a model for human adenocarcinomas, and especially for the pneumonic-type bronchioloalveolar cancer [18]. Similarly to ovine pulmonary adenocarcinoma, bronchioloalveolar cancer is a slow-growing tumour with rare distant metastases. It is clinically associated with highly productive cough and progressive restrictive respiratory failure $[18,20]$. The disease is usually sporadic within flocks but can be epizootic in some specific conditions of farming, leading to the death of up to $5 \%$ of the animals [16].
Given the similarities between ovine pulmonary adenocarcinoma and human BAC, a viral cause to human bronchioloalveolar cancer has long been hypothesized; but up to now reports exploring the link between JSRV and BAC [21-23] remain controversial. Nevertheless, ovine pulmonary adenocarcinoma offers an invaluable animal model for studying the molecular mechanisms of lung epithelial transformation occurring in cancer.

\section{JSRV AND ITS ONCOGENIC ENVELOPE}

JSRV genome contains the typical retrovirus genes gag, pro, pol and env respectively encoding the capsid and nucleocapsid proteins, the protease, the enzymatic activities and the envelope glycoproteins. An additional small ORF (open reading frame), called " $\mathrm{x}$ " and encoding a putative protein of unknown function, overlaps the end of the pol gene (Fig. 2). JSRV mainly infects and only transforms alveolar type II cells and Clara cells [24] leading to development of tumours in the deep lung. Using molecular detection of RNA and DNA, JSRV has been demonstrated to infect not only the respiratory tract but also several lymphoid tissues such as the mediastinal lymph nodes draining the lungs [25]. JSRV has been detected at low level in the spleen, the thymus, the bone marrow and the peripheral blood mononuclear cells [25]. Although very few cells of the immune systems are infected, the highest provirus load is in the monocytes/macrophages, followed by $\mathrm{B}$ and $\mathrm{T}$ cells. There is no evidence of virus transformation of any of these cell types $[25,26]$. Hyal2 (Hyaluronidase type 2) is the cell receptor for JSRV [27] and is expressed at the surface of many cell types. Hyal2 expression is not restricted to lung epithelial cells, suggesting that the entry of the virus into the cells is not the sole determinant of pathogenesis.

A family of related endogenous sequences, enJSRV closely related to the exogenous JSRV, is present in
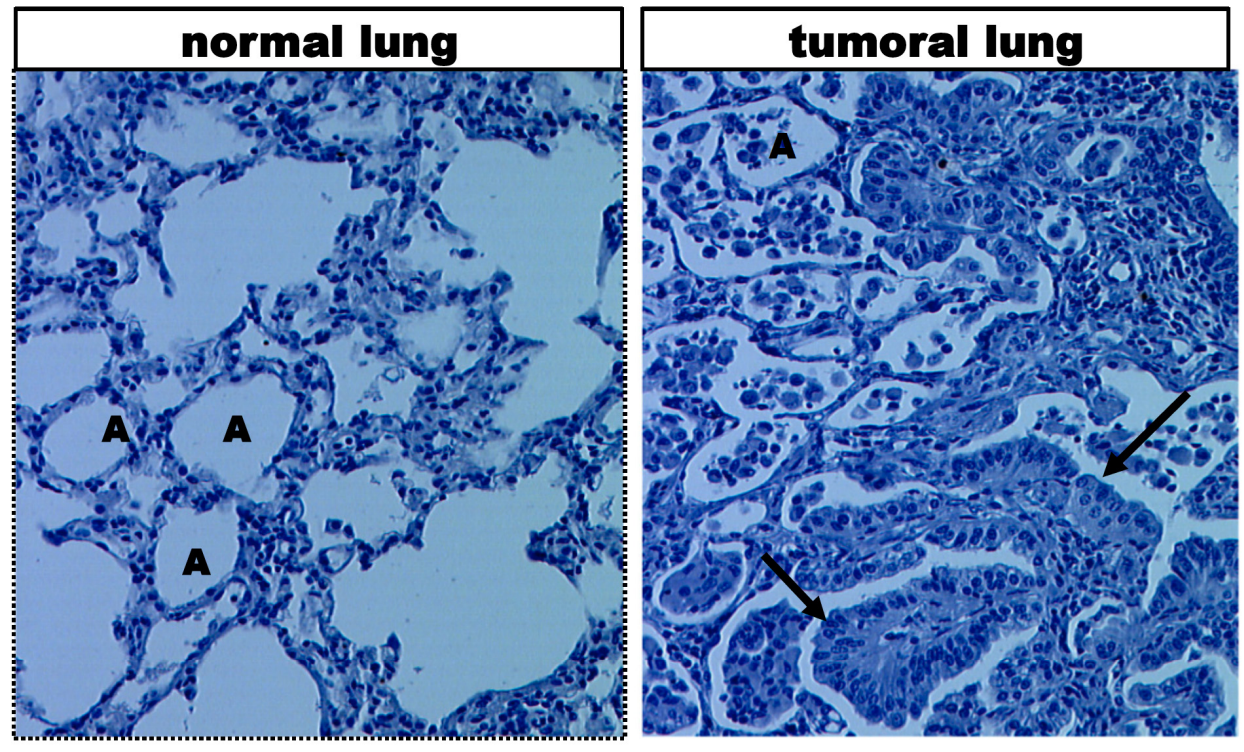

Fig. (1). Tumoral lesions observed during ovine pulmonary adenocarcinoma result from the transformation and accumulation of alveolar type II cells in the alveoli and Clara Cells in the bronchioli. While the normal lung presents large open alveoli (A), the architecture of the parenchyma is disturbed in the tumoral lung. Arrows point the accumulation of airway type II cells along the alveoli walls in a typical tumour. 

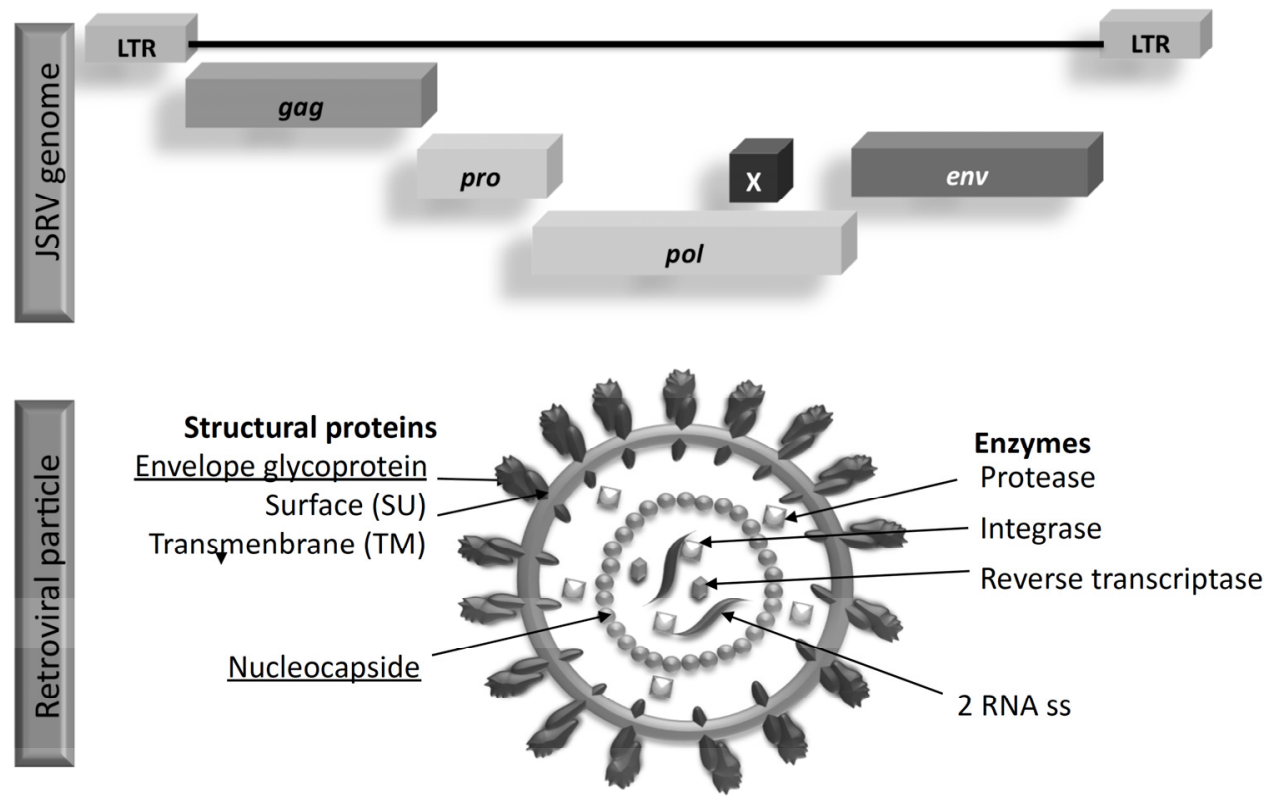

Fig. (2). JSRV viral genome is typically a dimmer of linear, positive-sense, single-stranded RNA with each strand being 7.5 kilobases in length. The gag gene encodes the matrix, the capsid and the nucleocapsid; the pro gene encodes a protease, the pol gene encodes the reverse transcriptase, RNaseH and integrase proteins; and the $\boldsymbol{e n v}$ gene encodes the glycoproteins needed for receptor recognition and envelope anchoring. An important feature of the retroviral genome is the long terminal repeat (LTR) regions found on both end of the provirus genome. The LTR plays an important role in initiating viral DNA synthesis and its integration as well as regulating transcription of the viral genes. The JSRV genome also contains an additional gene called "x" of unknown role.

domestic and wild ungulates [28]. JSRV and enJSRV genomes are highly related with $90-98 \%$ homology in deduced amino-acid sequences [29]. Endogenous JSRVs derive from ancestral integration of exogenous JSRV into the host genome. They are maintained as Mendelian genes and are vertically transferred to the offspring. They are defective for at least one viral gene as a result of mutations acquired during their integration into the germ-line. The involvement of enJSRV in the carcinogenesis remains unknown.

It is now clearly established that JSRV induces tumors via the oncogenic properties of its envelope, Env, which is both necessary and sufficient to induce transformation (reviewed in [30]). The oncogenic property of JSRV Env has been shown both in vitro in various cell types including murine NIH 3T3 fibroblasts [33], rat 208F fibroblasts [27], avian DF-1 fibroblasts [34, 35], bronchial human BEAS-2B epithelial cells [36], canine kidney MDCK epithelial cells [37], and rat kidney RK3E cells [38]; and in vivo in immunedeficient mice [31] and in sheep [32]. This oncogenic mechanism is unusual and JSRV is among the few retroviruses using one of its structural proteins to transform cells. Deletion experiments show that the TM (TransMembrane) region of the envelope is the main determinant for cell transformation [39-41]. The cytoplasmic tail (CT) of TM, composed of 43 amino acids, is essential for the transformation process in MDCK and NIH-3T3 cells [37, 42]. This region contains a peptidic YXXM motif, corresponding to a potential consensus site (phosphorylated on tyrosine $\mathrm{Y}$ ) linked to the $\mathrm{SH} 2$ domain of the p85 subunit of PI3K (Phosphatidyl-Inositol-3 Kinase), a kinase that activates Akt (Fig. 3). The mechanisms leading to JSRVinduced cell transformation are in fact much more complex than previously considered. Several experiments ruled out a direct role for the YXXM motif in Akt activation [35, 37, 43].

Although a number of points remain unanswered, the transformation steps are dependent on the nature and origin of the cells. Hence, understanding the key events in the transformation of ovine alveolar type II cells is now the challenge of ongoing work. Our group has developed primary cell cultures isolated from ovine tumoral lungs [44, 45]; those tumour-derived cells are alveolar type II cells as revealed by the presence of lamellar bodies in the cytoplasm, and the expression of surfactant protein C (SP-C) and A (SPA). We have reported activation of telomerase in ovine pulmonary adenocarcinoma-derived alveolar type II cells and in tumoral lung tissues, suggesting that replicative senescence may be negatively regulated in this tumour. Moreover, the Akt pathway deregulation has been shown to be deregulated in the tumoral process [45] (Fig. 3).

\section{ADAPTATIVE IMMUNE RESPONSE TO JSRV}

The immune response to JSRV infection is poorly understood [46]. The cell-mediated response is narrow with CD4+ lymphocytopenia and neutrophilia being demonstrated in the peripheral blood of adult sheep during the terminal stages of the cancer [26, 47]. More recently, a study reports invading macrophages in the tumours and its surrounding, during naturally or experimentally induced ovine pulmonary adenocarcinoma [48]. Immature macrophages (CD14+, weak $\mathrm{CD} 11 \mathrm{~b}+$ ) or monocytes expressing high levels of IFN- $\gamma$ are present in the immediate vicinity of the tumour and within the alveolar lumina. Macrophage activity seems to be suppressed at the periphery of the tumour, suggesting a local immune suppressive response that may be mediated by the 


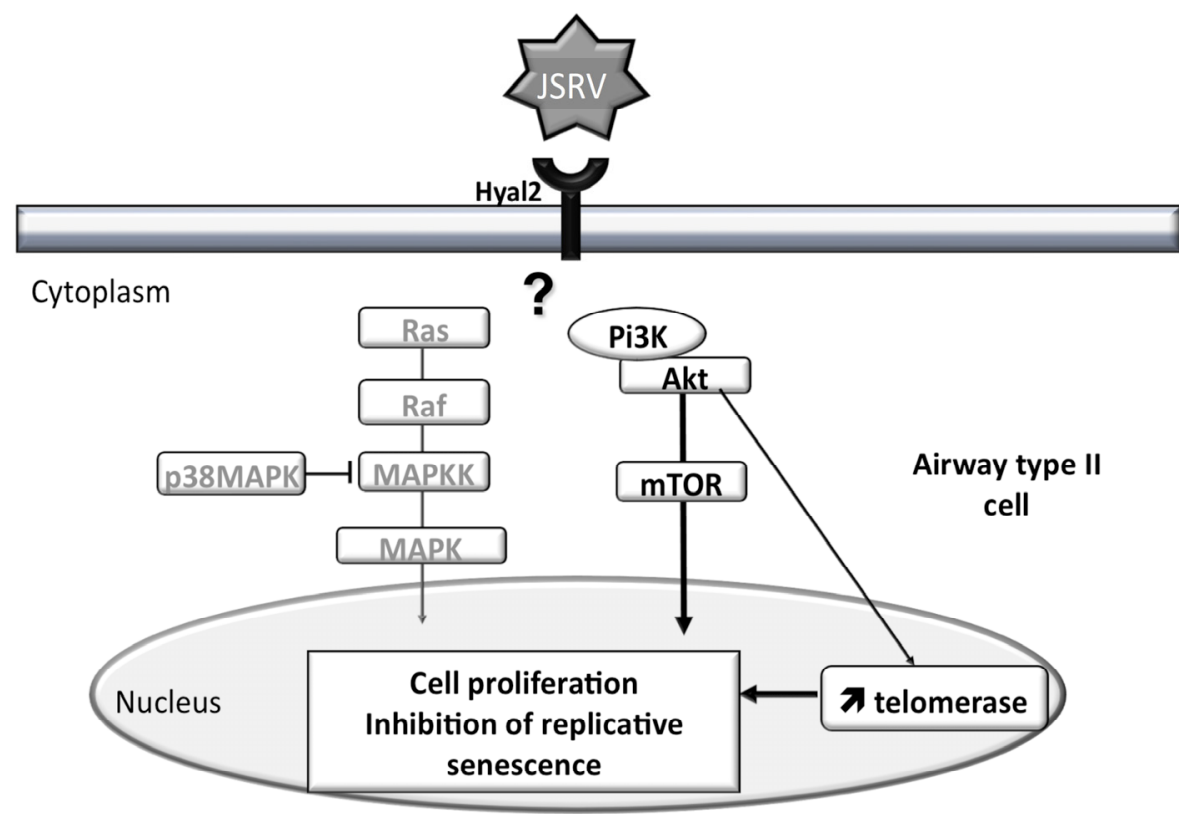

Fig. (3). Signalling pathways activated by JSRV in lung epithelial cells. Primary alveolar type II cells derived from ovine tumours display a proliferative advantage compared to normal cells, with a high telomerase activity leading to inhibition of the replicative senescence. Those cells also show a deregulation of the Pi3K/Akt pathway. These mechanisms are implicated in the tumour development and maintenance. Works done on various cell lines have shown that the MAPK pathways can also be implicated when cells are expressing the envelope protein of JSRV [30].

surfactant secretion [48]. No increase of dendritic, B and $\gamma \delta \mathrm{T}$ cell populations has been evidenced. Older reports suggested induction of a minor immunosuppression upon JSRV infection and its association with an increased susceptibility to other infectious agents such as Pasteurella haemolityca [49]. Reduced lymphoproliferative responses to mitogen stimulation such as concanavalin A has been shown in terminally ill adult sheep as well as in JSRV-inoculated lamb, prior to the onset of clinical disease [46] suggesting that JSRV may compromise the host cellular immune response.

Interestingly, DC-LAMP/CD208 (Dendritic CellLysosomal Associated Membrane Protein) is strongly expressed at the apical side of alveolar type II cells in lung tissues from JSRV-infected animals and human bronchioloalveolar cancer [50]. DC-LAMP/CD208 has been originally described as a molecule specifically expressed in mature dendritic cells at the limiting membrane of $\mathrm{MHC}$ Class II-containing intracellular compartments involved in the MHC class II peptide loading and transport to the cell surface $[51,52]$. In mouse, DC-LAMP/CD208 colocalizes with MHC class II molecules in lamellar bodies, intracytoplasmic organelles specific of alveolar type II cells [50]. This may link DC-LAMP to the controversial role of alveolar type II cells in MHC class II restricted antigen presentation. Alveolar type II cells constitutively express

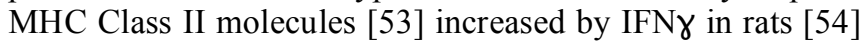
or pathological conditions in humans $[55,56]$, cathepsin [57] or CD54 [57]. Alveolar type II cells strategically localized at the interface between the parenchyma and the outside environment, express molecules required for efficient antigen presentation to $\mathrm{CD} 4+\mathrm{T}$ cells and may act as pulmonary antigen-presenting cells (APC) [58].
Evidence of a JSRV-specific humoral response remains a subject of discussion. Some studies showed a local IgA response, the formation of viral immune complexes, and a systemic antibody response that cross-react with recombinant antigens of highly related viruses [59-61]. On the other hand, accumulating data concluded to the absence of a JSRV-specific immune response [62-66]. This failure to detect JSRV specific antibodies in experimentally or naturally infected sheep remained puzzling. But more recently, it has been shown that antibodies could be detected in the serum or lung lavages of sheep immunized with recombinant JSRV capsid or surface glycoprotein inoculated with various adjuvants $[67,68]$. These results indicate that sheep are not inherently unresponsive to JSRV antigens and that the apparent tolerance, perhaps as a consequence of endogenous sequence expressed in utero or as a direct consequence of infection by JSRV, can be broken.

The infection of the immune system by JSRV may be advantageous to the virus either by directly facilitating the infection and the subsequent transformation of alveolar type II cells or indirectly by the induction of an immunosuppressive state. MMTV (mouse mammary tumour virus) and JSRV share interesting features. Both are retrovirus that have their own endogenous counterparts and are associated with epithelial tumours. Both viruses replicate actively in transformed epithelial cells but are also maintained at a low-level of infection in host lymphoid cells. However, the infection route and the targeted organs are distinct. MMTV enters through the digestive tract and has to reach the mammary gland, meaning that the infection of $B$ and $\mathrm{T}$ cells is an absolute requirement for the virus transfer to the target organs. In the case of JSRV, initial infection is mainly through the respiratory route and therefore alveolar type II cells and Clara cells are readily accessible. It is then 
conceivable that JSRV first infects lung epithelial cells and replicates. After few replications, surrounding lymphoid cells or phagocytic cells may carry the virus to the mediastinal lymph nodes. Holland and colleagues have shown that dissemination to the lymphoid tissues precedes tumour formation, as detection of JSRV proviral DNA is positive as early as 7 days post-inoculation and well before the onset of tumoral lesions [26]. Lymphocyte proliferation studies in response to mitogen stimulation have revealed an alteration in host cellular responses due to the presence of JSRV [46] (reduced response to ConA stimulation). This modification occurring early in the infection course is sustained through tumorigenesis up to the terminal stage of the disease. Lastly, despite low viral load in immune cells during the early steps post infection, the presence of viruses does alter the cell functions [46]. This may affect the function of lymphoid tissues and interfere or prevent the development of a specific immune response against the tumoral cells or the virus allowing the tumour progression (Fig. 4). At present, the role of JSRV infection of lymphoid tissues in pathogenesis is still unknown.

The majority of cases of JSRV-induced adenocarcinomas presents with a peripheral CD4+ lymphocytopenia and neutrophilia [26]. Taken together with the lack of circulating JSRV antibodies and the increased susceptibility to secondary bacterial infection, this could participate to a mild immunosuppression of JSRV-infected sheep. However, it is difficult to believe that immunosuppression could explain this absence of immune response, as even the highly immunosuppressive HIV is unable to abolish the circulation of virus-specific antibodies.

\section{enJSRV INTERFERENCE}

The absence of a strong immune JSRV-specific response may be related to the presence of the closely related enJSRV sequences. During ontogeny, clonal deletion of JSRV-specific repertoire may lead to immunological tolerance. Indeed, retroelements form a large and diverse family of mobile elements that can be found in most eukaryotic organisms. They propagate by reverse transcription of RNA intermediates and integrate their genetic information into the genomic DNA of the host cells. Retroelements include retrovirus-like elements, the integrated forms or proviruses consist of two long terminal repeats (LTRs) flanking an internal region containing one to three major open reading frame coding for structural (Gag and sometimes Env) and enzymatic (Pol) functions necessary for their replication cycle. These elements, known as endogenous retroviruses (ERVs) are transmitted through the germline as stable Mendelian genes. It is assumed that ERVs derived from ancient integration events of exogenous retroviruses into the germline. Generally, ERVs are transcriptionally silent and are often defective, differing from the exogenous counterparts by deletions or point mutations that render them unable to produce infectious virus. However, several ERVs maintain at least some intact open reading frames that can be expressed. Particularly, it has been shown that several ERVs containing gag and pol genes also retain the ability to encode functional Env glycoproteins.

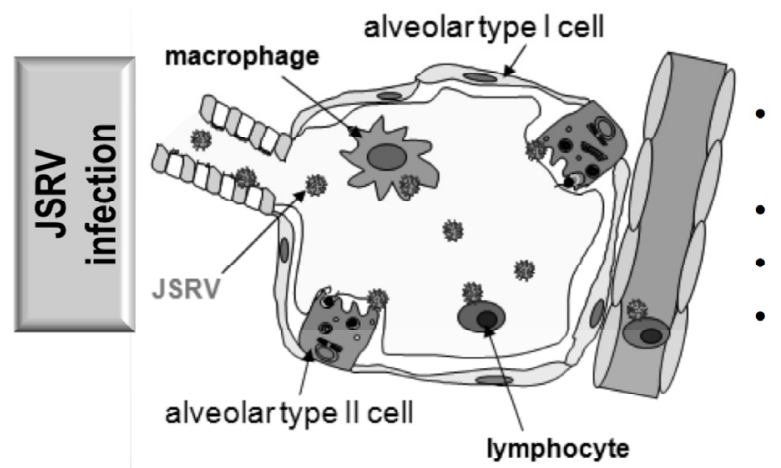

\section{EARLY STEPS}

Clara Cells and alveolar type II cells are infected by JSRV.

Macrophages, B and T cells : JSRV+/-

Detection of specific antibodies : low

- Reduced level of CD4+

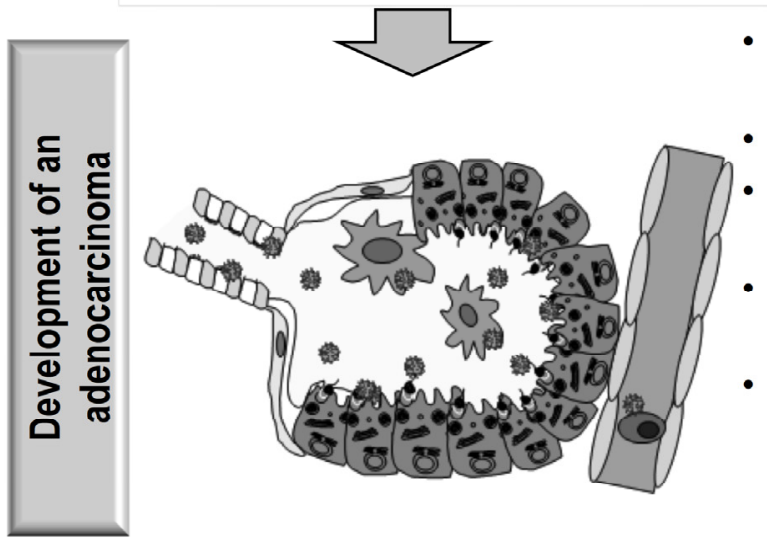

\section{LATE STAGE}

- Accumulation of transformed infected type II alveolar cells and Clara cell Lack of circulating JSRV antibodies. CD4+ lymphocytopenia and neutrophilia. No increase on dendritic cells

Increased susceptibility to secondary bacterial infection.

Local immune suppressive response. Invading macrophages in the tumour and its surroundings but no macrophage activity at the periphery of the tumour .

Fig. (4). Maintenance of a reduced cellular and humoral immune response upon JSRV infection and during the course of the virally-induced disease. At the early step of infection, a reduced level of CD4+, the absence of dendritic cells and a low level of circulating specific antibody may be observed. Later, while tumour has developed with alveolar type II cells colonizing the alveolar walls, there is a noticeable influx of macrophages around and in the tumour area, without any other major changes. 
The enJSRVs are genetically highly related to JSRV [69], which indicates a recent evolutionary relationship [70]. Several studies suggest that the endogenization of enJSRV occurred 20 My (millions years) in the ancestor species of ungulates [71]. The genomes of normal sheep and goats, as well as several other mammals, contain 15-20 copies of endogenous sequences related to JSRV [28, 72, 73]. Some of these enJSRV are transcriptionnally active in a wide range of tissues [25]. Tolerance may be acquired by expression of these sequences during in utero development and this may account for the low serological response to JSRV. Among the copies present in the sheep genome, one copy named enJS56A1 is a full-length provirus with complete open reading frame for $g a g$, pol and env. The enJS56A1 is mainly expressed in the placenta, but also at a lower level in the lung, the primary site of JSRV-replication. Interestingly, the enJS56A1 Gag can inhibit production of exogenous JSRV particles in a dominant manner [74]. This variant results from several amino acid replacements which are directly involved in the blocking of assembly of infectious JSRV particles. One explanation for the dominant negative effect of enJS56A1 Gag protein on particle release could be that this protein has a defective interaction with some component of the host cell sorting machinery that is responsible for transport and budding of JSRV. As a whole, the presence of this closely related endogenous version of JSRV could explain part of the tolerance and the low immunological response to infectious JSRV.

The immune response to JSRV is still in debate and needs more investigation to understand the mechanisms hold by this virus, leading to the development of a tumour in a poorly responding immunological environment. Whether this is due to the central tolerance related to endogenous forms or due to the peripheral tolerance induced by more direct JSRV immunosuppressive effects, any new therapeutic approach or vaccine development will have to deal with possible autoimmune inflammatory reactions or tumoral development.

\section{REFERENCES}

[1] Holt PG, Strickland DH, Wikstrom ME, Jahnsen FL. Regulation of immunological homeostasis in the respiratory tract. Nat Rev Immunol 2008; 8: 142-52.

[2] Holtzman MJ, Morton JD, Shornick LP, et al. Immunity, inflammation, and remodeling in the airway epithelial barrier: epithelial-viral-allergic paradigm. Physiol Rev 2002; 82: 19-46.

[3] Mayer AK, Dalpke AH. Regulation of local immunity by airway epithelial cells. Arch Immunol Ther Exp (Warsz) 2007; 55: 353-62.

[4] See H, Wark P. Innate immune response to viral infection of the lungs. Paediatr Respir Rev 2008; 9: 243-50.

[5] Mordstein M, Neugebauer E, Ditt V, et al. Lambda interferon renders epithelial cells of the respiratory and gastrointestinal tracts resistant to viral infections. J Virol 2010; 84: 5670-7.

[6] Randall RE, Goodbourn S. Interferons and viruses: an interplay between induction, signalling, antiviral responses and virus countermeasures. J Gen Virol 2008; 89: 1-47.

[7] Bals R, Hiemstra PS. Innate immunity in the lung: how epithelial cells fight against respiratory pathogens. Eur Respir J 2004; 23 : 327-33.

[8] Johnston SL. Overview of virus-induced airway disease. Proc Am Thorac Soc 2005; 2: 150-6.

[9] Mason RJ. Biology of alveolar type II cells. Respirology 2006; 11(Suppl): S12-5.

[10] Miura TA, Wang J, Holmes KV, Mason RJ. Rat coronaviruses infect rat alveolar type I epithelial cells and induce expression of CXC chemokines. Virology 2007; 369: 288-98.
[11] Wang J, Oberley-Deegan R, Wang S, et al. Differentiated human alveolar type II cells secrete antiviral IL-29 (IFN-lambda 1) in response to influenza A infection. J Immunol 2009; 182: 1296-304.

[12] Crouch EC. Surfactant protein-D and pulmonary host defense. Respir Res 2000; 1: 93-108.

[13] Doss M, White MR, Tecle T, et al. Interactions of alpha-, beta-, and theta-defensins with influenza A virus and surfactant protein D. J Immunol 2009; 182: 7878-87.

[14] Kishore U, Greenhough TJ, Waters P, et al. Surfactant proteins SPA and SP-D: structure, function and receptors. Mol Immunol 2006; 43: 1293-315.

[15] Klotman ME, Chang TL. Defensins in innate antiviral immunity. Nat Rev Immunol 2006; 6: 447-56.

[16] Griffiths DJ, Martineau HM, Cousens C. Pathology and pathogenesis of ovine pulmonary adenocarcinoma. J Comp Pathol 2010; 142: 260-83.

[17] Read WL, Page NC, Tierney RM, Piccirillo JF, Govindan, R. The epidemiology of bronchioloalveolar carcinoma over the past two decades: analysis of the SEER database. Lung Cancer 2004; 45: 137-42.

[18] Mornex JF, Thivolet F, De las Heras M, Leroux C. Pathology of human bronchioloalveolar carcinoma and its relationship to the ovine disease. Curr Top Microbiol Immunol 2003; 275: 225-48.

[19] Bonne C. Morphological resemblance of pulmonary adenomatosis (jaaksiekte) in sheep and certain cases of cancer of the lung in man. Am J Cancer 1939; 35: 491-501.

[20] Wislez M, Gounant V, Cadranel J. Bronchioloalveolar carcinoma. Rev Mal Respir 2005; 22: 8S70-5.

[21] De las Heras M, Barsky SH, Hasleton P, et al. Evidence for a protein related immunologically to the jaagsiekte sheep retrovirus in some human lung tumours. Eur Respir J 2000; 16: 330-2.

[22] Hiatt KM, Highsmith WE. Lack of DNA evidence for jaagsiekte sheep retrovirus in human bronchioloalveolar carcinoma. Hum Pathol 2002; 33: 680.

[23] Yousem SA, Finkelstein SD, Swalsky PA, Bakker A, Ohori N P. Absence of jaagsiekte sheep retrovirus DNA and RNA in bronchioloalveolar and conventional human pulmonary adenocarcinoma by PCR and RT-PCR analysis. Hum Pathol 2001; 32: $1039-42$

[24] Platt JA, Kraipowich N, Villafane F, DeMartini JC. Alveolar type II cells expressing jaagsiekte sheep retrovirus capsid protein and surfactant proteins are the predominant neoplastic cell type in ovine pulmonary adenocarcinoma. Vet Pathol 2002; 39: 341-52.

[25] Palmarini M, Holland MJ, Cousens C, Dalziel RG, Sharp JM. Jaagsiekte retrovirus establishes a disseminated infection of the lymphoid tissues of sheep affected by pulmonary adenomatosis. J Gen Virol 1996; 77( Pt 12): 2991-8.

[26] Holland MJ, Palmarini M, Garcia-Goti M, et al. Jaagsiekte retrovirus is widely distributed both in $\mathrm{T}$ and $\mathrm{B}$ lymphocytes and in mononuclear phagocytes of sheep with naturally and experimentally acquired pulmonary adenomatosis. J Virol 1999; 73: 4004-8.

[27] Rai SK, Duh FM, Vigdorovich V, Danilkovitch-Miagkova A, Lerman MI, Miller AD. Candidate tumor suppressor HYAL2 is a glycosylphosphatidylinositol (GPI)-anchored cell-surface receptor for jaagsiekte sheep retrovirus, the envelope protein of which mediates oncogenic transformation. Proc Natl Acad Sci U S A 2001; 98: 4443-8.

[28] Hecht SJ, Stedman KE, Carlson JO, DeMartini JC. Distribution of endogenous type $\mathrm{B}$ and type $\mathrm{D}$ sheep retrovirus sequences in ungulates and other mammals. Proc Natl Acad Sci U S A 1996; 93: 3297-302.

[29] Palmarini M, Hallwirth C, York D, et al. Molecular cloning and functional analysis of three type D endogenous retroviruses of sheep reveal a different cell tropism from that of the highly related exogenous jaagsiekte sheep retrovirus. J Virol 2000; 74: 8065-76.

[30] Leroux C, Girard N, Cottin V, Greenland T, Mornex JF, Archer F. Jaagsiekte Sheep Retrovirus (JSRV): from virus to lung cancer in sheep. Vet Res 2007; 38: 211-28.

[31] Wootton SK, Halbert CL, Miller AD. Sheep retrovirus structural protein induces lung tumours. Nature 2005; 434: 904-7.

[32] Caporale M, Cousens C, Centorame P, Pinoni C, De las Heras M, Palmarini M. Expression of the jaagsiekte sheep retrovirus envelope glycoprotein is sufficient to induce lung tumors in sheep. J Virol 2006; 80: 8030-7. 
[33] Maeda N, Palmarini M, Murgia C, Fan, H. Direct transformation of rodent fibroblasts by jaagsiekte sheep retrovirus DNA. Proc Natl Acad Sci U S A 2001; 98: 4449-54

[34] Allen TE, Sherrill KJ, Crispell SM, Perrott MR, Carlson JO, DeMartini JC. The jaagsiekte sheep retrovirus envelope gene induces transformation of the avian fibroblast cell line DF-1 but does not require a conserved $\mathrm{SH} 2$ binding domain. J Gen Virol 2002; 83: 2733-42.

[35] Zavala G, Pretto C, Chow YH, et al. Relevance of Akt phosphorylation in cell transformation induced by Jaagsiekte sheep retrovirus. Virology 2003; 312: 95-105.

[36] Danilkovitch-Miagkova A, Duh FM, Kuzmin I, et al. Hyaluronidase 2 negatively regulates RON receptor tyrosine kinase and mediates transformation of epithelial cells by jaagsiekte sheep retrovirus. Proc Natl Acad Sci U S A 2003; 100: 4580-5.

[37] Liu SL, Miller AD. Transformation of madin-darby canine kidney epithelial cells by sheep retrovirus envelope proteins. J Virol 2005; 79: 927-33.

[38] Maeda N, Fu W, Ortin A, de las Heras M, Fan H. Roles of the RasMEK-mitogen-activated protein kinase and phosphatidylinositol 3kinase-Akt-mTOR pathways in Jaagsiekte sheep retrovirus-induced transformation of rodent fibroblast and epithelial cell lines. J Virol 2005; 79: 4440-50.

[39] Chow YH, Alberti A, Mura M, et al. Transformation of rodent fibroblasts by the jaagsiekte sheep retrovirus envelope is receptor independent and does not require the surface domain. J Virol 2003; 77: 6341-50.

[40] Hofacre A, Fan H. Multiple domains of the Jaagsiekte sheep retrovirus envelope protein are required for transformation of rodent fibroblasts. J Virol 2004; 78: 10479-89.

[41] Hull S, Fan H. Mutational analysis of the cytoplasmic tail of jaagsiekte sheep retrovirus envelope protein. J Virol 2006; 80: 8069-80.

[42] Palmarini M, Maeda N, Murgia C, De-Fraja C, Hofacre A, Fan H. A phosphatidylinositol 3-kinase docking site in the cytoplasmic tail of the Jaagsiekte sheep retrovirus transmembrane protein is essential for envelope-induced transformation of NIH 3 T3 cells. J Virol 2001; 75: 11002-9.

[43] Liu SL, Lerman MI, Miller AD. Putative phosphatidylinositol 3kinase $(\mathrm{PI} 3 \mathrm{~K})$ binding motifs in ovine betaretrovirus Env proteins are not essential for rodent fibroblast transformation and PI3K/Akt activation. J Virol 2003; 77: 7924-35.

[44] Archer F, Jacquier E, Lyon M, et al. Alveolar type II cells isolated from pulmonary adenocarcinoma: a model for JSRV expression in vitro. Am J Respir Cell Mol Biol 2007; 36: 534-40.

[45] Suau F, Cottin V, Archer F, et al. Telomerase activation in a model of lung adenocarcinoma. Eur Respir J 2006; 27: 1175-82.

[46] Summers C, Neill W, Dewar P, et al. Systemic immune responses following infection with Jaagsiekte sheep retrovirus and in the terminal stages of ovine pulmonary adenocarcinoma. J Gen Virol 2002; 83: 1753-7.

[47] Rosadio RH, Sharp JM. Leukocyte frequency alterations in sheep with naturally and experimentally induced lung cancer. Veterinary Medicine 1992: 46-51.

[48] Summers C, Norval M, De Las Heras M, Gonzalez L, Sharp JM, Woods GM. An influx of macrophages is the predominant local immune response in ovine pulmonary adenocarcinoma. Vet Immunol Immunopathol 2005; 106: 285-94.

[49] Myer MS, Huchzermeyer HF, York DF, Hunter P, Verwoerd DW, Garnett HM. The possible involvement of immunosuppression caused by a lentivirus in the aetiology of jaagsiekte and pasteurellosis in sheep. Onderstepoort J Vet Res 1988; 55: 127-33.

[50] Salaun B, de Saint-Vis B, Pacheco N, et al. CD208/dendritic celllysosomal associated membrane protein is a marker of normal and transformed type II pneumocytes. Am J Pathol 2004; 164: 861-71.

[51] Barois N, de Saint-Vis B, Lebecque S, Geuze HJ, Kleijmeer MJ. MHC class II compartments in human dendritic cells undergo profound structural changes upon activation. Traffic 2002; 3: 894905.

[52] de Saint-Vis B, Vincent J, Vandenabeele S, et al. A novel lysosome-associated membrane glycoprotein, DC-LAMP, induced upon DC maturation, is transiently expressed in MHC class II compartment. Immunity 1998; 9: 325-36.
[53] Cunningham AC, Milne DS, Wilkes J, Dark JH, Tetley TD, Kirby JA. Constitutive expression of MHC and adhesion molecules by alveolar epithelial cells (type II pneumocytes) isolated from human lung and comparison with immunocytochemical findings. J Cell Sci 1994; 107 ( Pt 2): 443-9.

[54] Schneeberger EE, DeFerrari M, Skoskiewicz MJ, Russell PS, Colvin RB. Induction of MHC-determined antigens in the lung by interferon-gamma. Lab Invest 1986; 55: 138-44.

[55] Arbustini E, Morbini P, Diegoli M, et al. Coexpression of aspartic proteinases and human leukocyte antigen-DR in human transplanted lung. Am J Pathol 1994; 145: 310-21.

[56] Komatsu T, Yamamoto M, Shimokata K, Nagura H. Phenotypic characterization of alveolar capillary endothelial cells, alveolar epithelial cells and alveolar macrophages in patients with pulmonary fibrosis, with special reference to MHC class II antigens. Virchows Arch A Pathol Anat Histopathol 1989; 415: 7990 .

[57] Guzman J, Izumi T, Nagai S, Costabel U. ICAM-1 and integrin expression on isolated human alveolar type II pneumocytes. Eur Respir J 1994; 7: 736-9.

[58] Zissel G, Ernst M, Rabe K, et al. Human alveolar epithelial cells type II are capable of regulating $\mathrm{T}$-cell activity. J Investig Med 2000; 48: 66-75.

[59] Verwoerd DW, Payne AL, York DF, Myer MS. Isolation and preliminary characterization of the jaagsiekte retrovirus (JSRV). Onderstepoort J Vet Res 1983; 50: 309-16.

[60] Rosati S, Kwang J, Tolari F, Keen J. Characterization of enzootic nasal tumor virus capsid antigen. Vet Microbiol 1996; 53: 261-9.

[61] Kwang J, Rosati S, Yang S, Juste RA, de la Concha-Bermejillo A Recognition of ovine lentivirus gag gene products by serum from infected sheep. Vet Immunol Immunopathol 1996; 55: 107-14.

[62] Sharp JM, Herring AJ. Sheep pulmonary adenomatosis: demonstration of a protein which cross-reacts with the major core proteins of Mason-Pfizer monkey virus and mouse mammary tumour virus. J Gen Virol 1983; 64 (Pt 10): 2323-7.

[63] Demartini JC, Rosadio RH, Lairmore MD. The etiology and pathogenesis of ovine pulmonary carcinoma (sheep pulmonary adenomatosis). Vet Microbiol 1988; 17: 219-36.

[64] Verwoerd D W. In: Virus Infections of Ruminants. Dento Z Mosein B, Eds. Elsevier 1990; pp. 453-62.

[65] De las Heras M, Garcia de Jalon JA, Minguijon E, Gray EW, Dewar P, Sharp J M. Experimental transmission of enzootic intranasal tumors of goats. Vet Pathol 1995; 32: 19-23.

[66] Ortin A, Minguijon E, Dewar P, et al. Lack of a specific immune response against a recombinant capsid protein of Jaagsiekte sheep retrovirus in sheep and goats naturally affected by enzootic nasal tumour or sheep pulmonary adenomatosis. Vet Immunol Immunopathol 1998; 61: 229-37.

[67] Sharp JM, DeMartini JC. Natural history of JSRV in sheep. Curr Top Microbiol Immunol 2003; 275: 55-79.

[68] Summers C, Dewar P, van der Molen R, et al. Jaagsiekte sheep retrovirus-specific immune responses induced by vaccination: a comparison of immunisation strategies. Vaccine 2006; 24: 1821-9.

[69] DeMartini JC, Carlson JO, Leroux C, Spencer T, Palmarini M. Endogenous retroviruses related to jaagsiekte sheep retrovirus. Curr Top Microbiol Immunol 2003; 275: 117-37.

[70] Murcia PR, Arnaud F, Palmarini M. The transdominant endogenous retrovirus enJS56A1 associates with and blocks intracellular trafficking of Jaagsiekte sheep retrovirus Gag. J Virol 2007; 81: 1762-72.

[71] Dunlap KA, Palmarini M, Varela M, et al. Endogenous retroviruses regulate periimplantation placental growth and differentiation. Proc Natl Acad Sci U S A 2006; 103: 14390-5.

[72] York, D F, Vigne, R, Verwoerd, D W, Querat G. Nucleotide sequence of the jaagsiekte retrovirus, an exogenous and endogenous type D and B retrovirus of sheep and goats. J Virol 1992; 66: 4930-9.

[73] Carlson J, Lyon M, Bishop J, et al. Chromosomal distribution of endogenous Jaagsiekte sheep retrovirus proviral sequences in the sheep genome. J Virol 2003; 77: 9662-8.

[74] Palmarini M, Mura M, Spencer TE. Endogenous betaretroviruses of sheep: teaching new lessons in retroviral interference and adaptation. J Gen Virol 2004; 85: 1-13. 